\title{
Propriétés biochimiques et organoleptiques de trois mets traditionnels ivoiriens (attiéké, placali, attoukpou) à base de granulés de manioc natifs
}

\author{
Augustin Koffi YAO ${ }^{1}$, Djary Michel KOFFI ${ }^{2 *}$, Sika Hortense BLEI ${ }^{2}$, \\ Zaouli Bi IRIÉ ${ }^{1}$ et Sébastien Lamine NIAMKE ${ }^{2}$ \\ ${ }^{1}$ Laboratoire de Chimie-Technologie, Centre National de Recherche Agronomique (CNRA), BP 31 Bingerville, \\ Côte d'Ivoire. \\ ${ }^{2}$ Laboratoire de Biotechnologies, Filière Biochimie-Microbiologie de l'Unité de Formation et de Recherche \\ Biosciences de l'Université Félix Houphouët-Boigny, 22 BP 582 Abidjan, Côte d'Ivoire. \\ ${ }^{*}$ Corresponding author; E-mail: djaryss@yahoo.fr ; Tel: (225) 07028006
}

\section{RESUME}

Des granulés de manioc natif ont été produits et conservés pendant 24 mois en vu de les utiliser après reconstitution pour préparer les mets traditionnels. Trois mets traditionnels ivoiriens (attiéké, attoukpou, placali) ont été confectionnés avec les granulés de manioc natif produits à partir trois cultivars de manioc: Bonoua, Soglo et Yacé. Les propriétés biochimiques et organoleptiques de ces mets ont été déterminées selon les méthodes d'analyse standard. Les résultats ont montré que l'attiéké de manioc frais contenait 45,17\% d'amidon et $0,49 \%$ de sucres, tandis que celui obtenu avec les granulés avait une teneur en amidon de 31,45 et $8,4 \%$ de sucres. En ce qui concerne l'attoukpou, les teneurs en amidon et en sucres étaient respectivement de 47,93 et $0,8 \%$ lorsque le manioc frais est utilisé pour la préparation alors que les teneurs obtenues pour l'attoukpou préparé avec les granulés étaient de 35,5\% pour l'amidon et 8,4\% pour les sucres, comme dans le cas de l'attiéké des granulés. L'acidité titrable du placali est de 20 méq.g/100 g avec le manioc frais contre 5 méq. $\mathrm{g} / 100 \mathrm{~g}$, pour le produit obtenu avec les granulés. Le placali de manioc frais contient $0,9 \mathrm{mg} \mathrm{HCN} / 100 \mathrm{~g}$ et celui obtenu à partir des granulés n'en contient que des traces. Les teneurs en amidon, en sucres totaux et en sucres réducteurs sont respectivement de $8,37,12$, 3, et 3,03\% pour le placali de manioc frais contre $6,13,2$ et $3,55 \%$ pour celui fait à partir des granulés. L'évaluation des propriétés organoleptiques des produits a révélé qu'il n'y aucune différence significative entre les produits issus du manioc frais et ceux obtenus avec les granulés de manioc natif conservés pendant deux ans, s'agissant de la couleur, l'arôme et de la texture. Par contre, en ce qui concerne le goût et l'aigreur, les mets confectionnés avec les granulés de manioc natifs ont été jugés meilleurs par rapport à ceux issus du manioc frais.

(C) 2015 International Formulae Group. All rights reserved.

Mots clés : Granulés de manioc natifs, propriétés biochimiques et organoleptiques, mets traditionnels ivoiriens, attiéké, placali, attoukpou.

\section{INTRODUCTION}

Le manioc est une culture vivrière importante par sa production et sa consommation. Au plan mondial, il occupe, le $2^{\text {ème }}$ rang des productions vivrières, avec des productions annuelles estimées à 242.000 .000 tonnes (FAO, 2009a). Le manioc est le troisième produit alimentaire dans les régions 
tropicales après le riz et le maïs; il reste la nourriture de base de plus de 500 millions de personnes (FAO, 2008). Le manioc est utilisé aussi bien pour l'alimentation humaine et animale et pour la fabrication des produits industriels. En Afrique, la production annuelle de manioc est estimée à 121500000 tonnes. Le Nigeria est le premier producteur mondial de manioc avec des productions de 44582 000 tonnes (FAO, 2009 b).

En Côte d'Ivoire, le manioc est une denrée qui est produite sur environ 4/5 du territoire national (N'Zué et al., 2004). Il est souvent cultivé en association avec l'igname, mais des plantations de manioc en culture pure existent dans les zones de production. La production annuelle estimée à 2.951.156 tonnes, occupe, le $2^{\text {ème }}$ rang des productions vivrières, après l'igname (Du Croquet, 2002 ; Anonyme, 2008), est disponible toute l'année.

Les racines tubéreuses de manioc ont une courte durée de conservation à température ambiante. Après la récolte, elles ont une durée de vie maximale de 3 jours audelà desquels le noircissement et les pourritures apparaîssent, les rendant impropres à l'alimentation humaine, première utilisation de ce produit en Côte d'Ivoire (Yao et al., 2006; Onwuka and Ogbogu, 2007 ; Udedible et al., 2009 ). Les racines épluchées se détériorent plus rapidement que celles recouvertes de peau; la dégradation intervient quelques heures seulement après l'épluchage. La racine change de couleur, signe caractéristique des réactions d'oxydation, certainement, dues à la présence des polyphénols. Le trempage dans l'eau retarde ce processus de quelques heures, mais le séjour prolongé des racines épluchées dans l'eau entraîne la fermentation et le ramollissement, comme pendant le rouissage (Obadina et al., 2009).

En vu de résoudre le problème de la conservation $\mathrm{du}$ manioc, une méthode permettant de produire des granulés de manioc natif conservable sur deux ans a été mise au point (Yao, 2015). Dans la présente étude, ces granulés ont été utilisés pour confectionner des mets traditionnels (attiéké, attoukpou, placali) après reconstitution de la pâte de manioc frais broyé par réhydratation des granulés. Le présent article rend compte des résultats obtenus.

\section{MATERIEL ET METHODES \\ Matériel végétal}

Nous avons utilisé les cultivars Bonoua, Yacé et Soglo récoltés dans la région de Bingerville à $15 \mathrm{~km}$ d'Abidjan (Côte d'Ivoire). Les racines étaient âgées de 10 à 13 mois, comme recommandé par Nzué et al. (2004).

\section{Reconstitution de la pâte de manioc à partir des granulés}

Des tests de reconstitution des granulés pour avoir l'équivalent de manioc frais broyé, ont été faits pour déterminer la quantité d'eau nécessaire à mettre en œuvre. Ces produits réhydratés ont été ensemencés avec du ferment traditionnel (magnan) obtenu avec du manioc bouilli ou braisé et laissé fermenter pendant 3 jours sous atmosphère confinée dans des sachets plastiques. L'ensemencement se fait avec le ferment traditionnel finement broyé puis incorporé à la pâte de manioc et le tout est soigneusement mélangé. La pâte ainsi ensemencée est emballée dans des sacs de jute et pressée après $12 \mathrm{~h}$ de fermentation puis utilisées pour préparer l'attiéké, l'attoukpou et le placali. Le placali se prépare par chauffage de la pâte de manioc fermentée dans une marmite tout en remuant avec une spatule en bois jusqu'à l'obtention d'une pâte translucide. L'attiéké et l'attoukpou sont obtenus par cuisson à la vapeur des semoules de manioc obtenues après fermentation, pressage, granulation. Pour la semoule destinée à la confection de l'attiéké, une étape supplémentaire de séchage est nécessaire avant la cuisson.

\section{Tests organoleptiques}

L'évaluation des caractéristiques organoleptiques a été faite par 50 dégustateurs-consommateurs habituels de ces mets en notant de 1 à 5 les critères d'appréciation que sont : la couleur, le goût et 
l'aigreur, la texture, la tendreté et l'arôme, ce qui a permis de comparer les produits obtenus à ceux confectionnés avec le manioc frais.

\section{Analyses biochimiques}

\section{Détermination du taux d'humidité}

Selon la méthode AOAC (2000), les échantillons sont pesés $\left(\mathrm{P}_{0}\right)$ à l'aide d'une balance de précision de type SARTORUIS BP 310S, Gottingen, West Germany. Ils ont été séchés dans une étuve de marque (MEMERT, Schwabach West Germany), à $105^{\circ} \mathrm{C}$ pendant $24 \mathrm{~h}$. A la sortie de l'étuve, les échantillons ont été refroidis dans un dessiccateur et pesés $\left(\mathrm{P}_{1}\right)$; le pourcentage d'humidité a été déterminé par calcul selon, la formule :

$$
H=\frac{P_{0}-P_{1}}{P_{0}} \times 100
$$

\section{Détermination du taux de cendres}

Selon la méthode AOAC (2000), cette détermination a été faite par minéralisation d'un échantillon de $5 \mathrm{~g}\left(\mathrm{P}_{0}\right)$ à $550{ }^{\circ} \mathrm{C}$ pendant $6 \mathrm{~h}$ dans un four à moufle (NABERTERM, Gmbh LT9/11/B180, Germany), jusqu'à destruction de toutes les matières organiques contenus dans l'échantillon. La pesée $\left(\mathrm{P}_{1}\right)$ après refroidissement dans un dessiccateur de la cendre obtenue a permis de déterminer le taux de cendres selon la formule :

$\%$ Cendres $=\frac{\mathrm{P}_{0}-\mathrm{P}_{1}}{\mathrm{P}_{0}} \times 100$

\section{Dosage de l'acidité titrable}

Selon la méthode AOAC (2000), ce dosage consiste à mesurer l'acidité titrable d'un produit avec une solution d'hydroxyde de sodium $(\mathrm{NaOH})$, de normalité $0,1 \mathrm{~N}$, en présence de phénolphtaléine comme indicateur coloré. Pour ce faire, une masse $\mathrm{P}$ de $10 \mathrm{~g}$ d'un échantillon de produit finement broyé est délayée dans $75 \mathrm{~mL}$ d'eau distillée, puis laissé macérer et le surnageant est filtré; 3 gouttes de phénolphtaléines sont ajoutées à un volume $\mathrm{V}_{1}=10 \mathrm{~mL}$ de ce filtrat et le dosage est effectué en versant la solution de $\mathrm{NaOH}$ de normalité $\mathrm{N}_{2} \quad(0,1 \mathrm{~N})$ jusqu'à l'apparition d'une couleur rose. Soit $\mathrm{V}_{2}$ le volume de la solution de $\mathrm{NaOH}$ versé, la normalité $\mathrm{N}_{1}$ du filtrat prélevé est obtenue par la formule :

$$
\mathrm{N}_{1}=\frac{\mathrm{N}_{2} \times \mathrm{V}_{2}}{\mathrm{~V}_{1}}
$$

Cette normalité a été convertie en milliéquivalent pour $100 \mathrm{~g}$ d'échantillon frais (méq/100g) en utilisant la formule (5).

Acidité (méq.g/100 g) $=\frac{\mathrm{N}_{1} \times 10^{5}}{\mathrm{P}}$

L'acidité en méq.g pour $100 \mathrm{~g}$ de matière fraîche peut aussi être exprimée en équivalent acide lactique pour $100 \mathrm{~g}$ en multipliant la valeur obtenue par 0,09 qui est la masse en $\mathrm{g}$ d'un milliéquivalent d'acide lactique.

Equivalent acide lactique $/ 100 \mathrm{~g}=$ Acidité (méq.g/100g) x 0,09

\section{Détermination du $\mathrm{pH}$}

La détermination du $\mathrm{pH}$ se fait en immergeant l'électrode de verre du $\mathrm{pH}$-mètre pré-étalonnée dans $10 \mathrm{~mL}$ de surnageant obtenu après macération de $10 \mathrm{~g}$ d'échantillon, dans $75 \mathrm{~mL}$ d'eau distillée. La valeur du $\mathrm{pH}$ affichée sur l'écran du $\mathrm{pH}$-mètre est relevée.

\section{Détermination du taux de matières grasses}

Cette détermination est basée sur le fait que les matières grasses sont solubles dans les solvants organiques tels que l'éther de pétrole et l'hexane. La détermination a été faite suivant la méthode du BIPEA (1976), consistant à extraire les matières grasses avec l'hexane qui est ensuite évaporé et le résidu séché puis pesé.

\section{Détermination de la valeur énergétique}

$\mathrm{La}$ valeur énergétique a été déterminée par calcul, selon la formule de Coleman (1970) utilisant les coefficients d'Atwater et Rosa (1899).

$\mathrm{E}$ (calories $)=(4 \times \%$ protéines $)+(4 \times \%$ glucides totaux $)+(9 \times \%$ lipides $)$ 


\section{Dosage des protéines brutes}

Le dosage des protéines a été fait selon la méthode BIPEA (1976) utilisant un distillateur Kjeldahl ; cette méthode est basée sur le dosage de l'azote total, qui est ensuite converti en taux de protéines. La minéralisation de $1 \mathrm{~g}$ d'échantillon des produits concernés se fait en présence de catalyseur composé de sulfate de cuivre $\left(\mathrm{CuSO}_{4)}\right.$ et de sulfate de potassium $\left(\mathrm{K}_{2} \mathrm{SO}_{4}\right)$ dans un digesteur type Buchi 430 (Digestor Germany), pendant 3 h. Ensuite, une distillation est effectuée dans un distillateur type Buchi 320, Germany, après addition de $10 \mathrm{~mL}$ de solution de soude $(\mathrm{NaOH})$ à $40 \%$ au minéralisat. Le distillat est recueilli dans une solution tampon d'acide borique préparée par dissolution de $10 \mathrm{~g}$ d'acide borique dans $1000 \mathrm{~mL}$ d'eau distillée et $11 \mathrm{~mL}$ de mélange d'indicateur coloré constitué de vert de bromocrésol et de rouge de méthyl. Le titrage $\mathrm{du}$ distillat se fait avec l'acide chlorhydrique ( $\mathrm{HCl})$ 0,1 N. Les taux d'azote total et de protéines brutes sont obtenus avec des formules:

Taux d'azote total $=$

$\mathrm{V}(\mathrm{HCl}) \times \mathrm{N}(\mathrm{HCl}) \times 0,014 \times 100$

\section{$\mathrm{P}$}

$\mathrm{V}(\mathrm{HCl})=$ volume $\mathrm{HCl}$ de la chute de burette $;$ $\mathrm{N}(\mathrm{HCl})=$ normalité de $\mathrm{HCl} ; 0,014$ $=$ coefficient affecté à la concentration de la solution normale d'azote $(14 / 1000) ; \mathrm{P}=$ poids de l'échantillon

Taux de protéines brutes $=$ Taux d'azote total $\times 6,25$

\section{Dosage de l'acide cyanhydrique}

Ce dosage a été effectué selon la méthode de Liebig-Denige (1971). $20 \mathrm{~g}$ d'échantillon ont été macérés dans $200 \mathrm{~cm}^{3}$ d'eau distillée pendant 3 à $4 \mathrm{~h}$, suivi de la distillation pour recueillir le distillat sur 20 $\mathrm{cm}^{3}$ de solution de soude contenant $0,5 \mathrm{~g}$ de soude. Ensuite, $100 \mathrm{~cm}^{3}$ du distillat sont prélevés et $8 \mathrm{~cm}^{3}$ d'iodure de potassium (KI) à $5 \%$ y sont ajoutés. Le dosage se fait avec une solution de nitrate d'argent $\left(\mathrm{AgNO}_{3} 0,02 \mathrm{~N}\right)$. Selon cette méthode, l'ion $\mathrm{CN}^{-}$tend à se combiner avec l'ion $\mathrm{Ag}^{+}$, pour former un précipité de cyanure d'argent $\mathrm{AgCN}$. Ce précipité ne se forme pas dans un milieu ammoniacal. La fin de la réaction est obtenue lorsque tous les ions $\mathrm{CN}^{-}$ont réagi; l'ion $\mathrm{Ag}^{+}$se combine avec les ions I' de l'indicateur iodure de potassium (KI) et le nitrate d'argent $\left(\mathrm{AgNO}_{3}\right)$ rend la solution opalescente. Soit $\mathbf{A}$ la quantité de $\mathrm{AgNO}_{3}$ en mL obtenue en fin de dosage, la quantité d'acide cyanhydrique (HCN) en mg d'échantillon est obtenue par la formule :

$$
\text { A } x 1,08=m g \text { HCN }
$$

\section{( $1 \mathrm{~mL} \mathrm{AgNO}_{3}$ équivaut à $1,08 \mathrm{mg} \mathrm{HCN}$ )}

\section{Détermination de la teneur en sucres totaux}

Les sucres totaux ont été dosés selon la méthode de Dubois (1956) utilisant l'acide sulfurique et le phénol. Elle consiste à déterminer les concentrations en sucres totaux de $0,5 \mathrm{~g}$ d'échantillon, sur une courbe étalon tracée en fonction de la concentration et de la densité optique de solutions de glucose de concentrations connues. Le spectromètre de marque THERMO SCIENTIFIC, type Hélios Oméga UV-VIS (USA) a été utilisé pour la détermination des densités optiques des solutions de sucres de concentrations inconnues. Ces densités optiques, rapportées sur la courbe étalon, permettent de déterminer la concentration en sucres totaux des échantillons.

\section{Détermination de la teneur en sucres réducteurs}

Les sucres réducteurs ont été dosés selon la méthode de Bernfeld (1955) utilisant l'acide 3,5-dinitrosalicylique (DNS). Elle consiste à déterminer les concentrations en sucres réducteurs de $0,5 \mathrm{~g}$ d'échantillon après extraction et purification, sur une courbe étalon tracée en fonction de la concentration et de la densité optique de solutions de glucose de concentrations connues. Le spectromètre de marque THERMO SCIENTIFIC, type Hélios Oméga UV-VIS (USA) a été utilisé pour la détermination des densités optiques à $546 \mathrm{~nm}$ des solutions de sucres de concentrations inconnues. Ces densités 
optiques, rapportées sur la courbe étalon, permettent de déterminer la concentration en sucres réducteurs des échantillons.

Détermination de la teneur en glucides totaux et en amidon

Le taux de glucides totaux et d'amidon ont été déterminés selon les formules décrites par Bertrand et Thomas (1910) :

Glucides totaux $(\%)=100-(\%$ humidité

$+\%$ protéines $+\%$ lipides $+\%$ cendres $)$

Taux d'amidon $=0,9(\%$ glucides totaux $\%$ sucres totaux)

\section{Analyse statistique des résultats}

Les résultats ont été soumis à des analyses de variances (ANOVA) réalisées avec le logiciel Stastica 7.0 en vue de comparer les moyennes. En cas de différence significative, le test de Newman-Keuls a permis d'identifier les moyennes responsables de la différence observée au seuil de 5\%.

\section{RESULTATS}

Reconstitution de la pâte et préparation des produits dérivés du manioc

Les granulés de manioc natif ont été reconstitués par réhydratation, avec de l'eau propre. Les essais de réhydratation des granulés ont permis de déterminer qu'il faut $1000 \mathrm{~g}$ d'eau (soit $1 \mathrm{~L}$ d'eau) pour la reconstitution de $1000 \mathrm{~g}$ de granulés de manioc natif afin d'obtenir l'équivalent de manioc frais broyé utilisé pour la préparation de l'attiéké et du placali. Pour la préparation de l'attoukpou, nous avons déterminé qu'il faut $800 \mathrm{~g}$ d'eau pour réhydrater $1000 \mathrm{~g}$ de granulés. Les produits obtenus sont présentés sur la Figure 1.

\section{Caractéristiques des produits obtenus}

Composition biochimique de l'attiéké et de l'attoukpou préparés à partir des granulés de manioc natif

Les résultats des analyses physicochimiques de l'attiéké et de l'attoukpou, sont présentés dans les Tableaux 1 et 2. Ces résultats montrent que les valeurs de l'humidité, des matières sèches, des glucides totaux et des matières grasses sont identiques pour ces deux produits, préparés, tant avec le manioc frais qu'avec les granulés de manioc natif reconstitués. Pour les teneurs en amidon et en sucres, des différences significatives ont été observées. L'attiéké de manioc frais contient $45,17 \%$ d'amidon et $0,49 \%$ de sucres, tandis que celui obtenu avec les granulés a une teneur en amidon de $31,45 \%$ et $8,4 \%$ de sucres. En ce qui concerne l'attoukpou, les teneurs en amidon et en sucres sont respectivement de $47,93 \%$ et $0,8 \%$ lorsque le manioc frais est utilisé pour la préparation alors que les teneurs obtenues pour l'attoukpou préparé avec les granulés sont de $35,5 \%$, pour l'amidon, et $8,4 \%$ pour les sucres comme dans le cas de l'attiéké des granulés.

Aucune différence significative n'a été notée pour le taux de protéines de l'attiéké et de l'attoukpou, préparés avec le manioc frais et avec les granulés de manioc natif (environ $1,5 \%)$. Des différences significatives ont été observées pour les teneurs en cellulose et en cendres ainsi que pour le $\mathrm{pH}$ et l'acidité titrable. Les valeurs obtenues pour l'attiéké varient de 1,2 à 1,5 pour les cendres et de 0,25 à 0,31 pour les celluloses. En ce qui concerne le $\mathrm{pH}$ et l'acidité titrable, les valeurs sont comprises respectivement entre 6,46 et 6,55 et 3,46 et 4,67 . Quant à l'attoukpou, ces valeurs vont respectivement de 0,25 à 2,$1 ; 1,2$ à 1,5 ; 5,46 à 6,55 et 3,46 à 4,67 pour les celluloses, les cendres, le pH et l'acidité triturable. Nous avons aussi noté une absence d'acide cyanhydrique (HCN) dans l'attiéké et l'attoukpou faits avec les granulés de manioc natif, alors que des valeurs de l'ordre de 0,47 $\mathrm{mg} / 100 \mathrm{~g}$ ont été obtenues pour les mêmes produits préparés à partir du manioc frais. L'énergie fournie par la consommation de 100 g d'attiéké et d'attoukpou est de l'ordre de $189,65 \mathrm{kcal}$ et est identique pour ces produits faits avec le manioc frais qu'avec les granulés.

Composition biochimique du placali préparé à partir des granulés de manioc natif et de manioc frais

La composition biochimique du placali préparé avec le manioc frais et avec les granulés, après conservation pendant $6 ; 12$; 
18 et 24 mois, est présentée dans le Tableau 3. Les résultats des analyses du placali ont montré qu'il contient $70 \%$ d'eau et $30 \%$ de matières sèches. Les glucides totaux représentent $22,74 \%$ et sont constitués de 12,3 à $13,5 \%$ de sucres totaux, dont $3 \%$ de sucres réducteurs et $8,37 \%$ d'amidon. Un taux de protéines de $4 \%$ et une absence de matière grasse ont été observés dans les deux types de placali. Le pH du placali obtenu à partir des granulés est de 6,7 et celui à partir du manioc frais est de 5,7. L'acidité titrable est de 20 méq.g/100 g avec le manioc frais contre 5 méq. g/100 g, pour le produit obtenu avec les granulés. Le placali de manioc frais contient $0,9 \mathrm{mg} \mathrm{HCN} / 100 \mathrm{~g}$ et celui des obtenu à partir des granulés n'en contient que des traces de HCN. La valeur énergétique est la même pour tous les types de placali, soit 106,96 kcal/100 g.
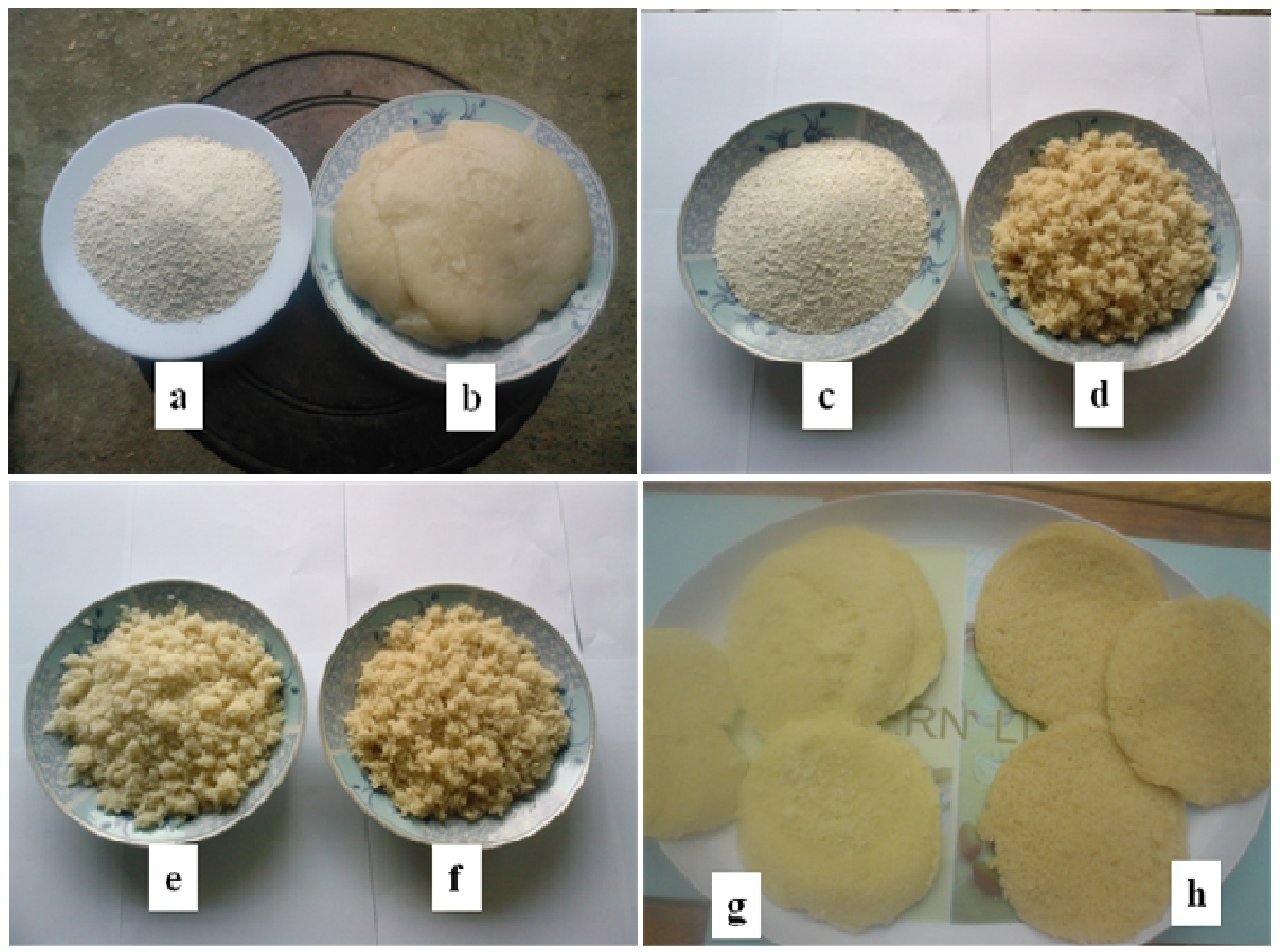

\section{Evaluation de la qualité organoleptique des produits}

Les Tableaux 4, 5 et 6 présentent les résultats de l'évaluation des caractères organoleptiques de l'attiéké, de l'attoukpou et du placali préparés avec le manioc frais et avec les granulés de manioc natif. L'analyse de ces résultats a montré des différences significatives entre les produits préparés avec le manioc frais et ceux préparés avec les granulés pour le critère goût et aigreur. Pour tous les autres critères (couleur, arôme, tendreté et texture) aucune différence significative n'a été observée. Ces produits ont été jugés identiques à ceux faits avec le manioc frais. Dans l'ensemble, les produits préparés avec les granulés ont obtenu une bonne appréciation des dégustateurs.

Figure 1: Mets traditionnel obtenus avec les granulés de manioc natif conservés pendant 24 mois. a et c: granulés de manioc natif ; b: placali; $\mathrm{d}$ et $\mathrm{f}$ : attiéké de granulés; $\mathrm{e}:$ attiéké de manioc frais ; $\mathrm{g}$ : attoukpou de manioc frais ; h : attoupkou de granulés.

\section{frais ; : attoupkou de granules.}


Tableau 1: Composition biochimique de l'attiéké préparé avec le manioc frais et avec les granulés de manioc natif (g/100 g de matière sèche).

\begin{tabular}{|c|c|c|c|c|c|c|}
\hline Paramètres & $\begin{array}{c}\text { Attiéké de } \\
\text { manioc frais }\end{array}$ & $\begin{array}{c}\text { Attiéké de } \\
\text { granulés non } \\
\text { conservés }\end{array}$ & $\begin{array}{c}\text { Attiéké de granulés } \\
\text { conservés pendant } \\
6 \text { mois }\end{array}$ & $\begin{array}{c}\text { Attiéké de granulés } \\
\text { conservés pendant } \\
12 \text { mois }\end{array}$ & $\begin{array}{c}\text { Attiéké de granulés } \\
\text { conservés pendant } \\
18 \text { mois }\end{array}$ & $\begin{array}{c}\text { Attiéké de granulés } \\
\text { conservés pendant } \\
24 \text { mois }\end{array}$ \\
\hline Humidité (\%) & $48,2 \pm 0,90^{\mathrm{a}}$ & $48,85 \pm 0,9^{\mathrm{a}}$ & $48,85 \pm 0,8^{\mathrm{a}}$ & $48,85 \pm 0,7^{\mathrm{a}}$ & $48,85 \pm 0,9^{\mathrm{a}}$ & $48,85 \pm 0,8^{\mathrm{a}}$ \\
\hline Matières sèches (\%) & $51,8 \pm 0,85^{\mathrm{a}}$ & $51,15 \pm 0,7^{\mathrm{a}}$ & $51,15 \pm 0,6^{\mathrm{a}}$ & $51,15 \pm 0,5^{\mathrm{a}}$ & $51,15 \pm 0,8^{\mathrm{a}}$ & $51,15 \pm 0,7^{\mathrm{a}}$ \\
\hline Matières grasses (\%) & $0,2 \pm 0,01^{\mathrm{a}}$ & $0,25 \pm 0,01^{\mathrm{a}}$ & $0,25 \pm 0,02^{\mathrm{a}}$ & $0,25 \pm 0,02^{\mathrm{a}}$ & $0,25 \pm 0,02^{\mathrm{a}}$ & $0,25 \pm 0,02^{\mathrm{a}}$ \\
\hline Glucides totaux (\%) & $49,13 \pm 0,7^{\mathrm{a}}$ & $49,5 \pm 0,6^{\mathrm{a}}$ & $49,5 \pm 0,5^{\mathrm{a}}$ & $49,5 \pm 0,5^{\mathrm{a}}$ & $49,5 \pm 0,6^{\mathrm{a}}$ & $49,5 \pm 0,5^{\mathrm{a}}$ \\
\hline Sucres totaux $(\%)$ & $0,8 \pm 0,01^{\mathrm{a}}$ & $8,4 \pm 0,1^{\mathrm{b}}$ & $8,4 \pm 0,1^{\mathrm{b}}$ & $8,4 \pm 0,2^{\mathrm{b}}$ & $8,4 \pm 0,1^{\mathrm{b}}$ & $8,4 \pm 0,2^{\mathrm{b}}$ \\
\hline Sucres réducteur $(\%)$ & $0,4 \pm 0,01^{\mathrm{a}}$ & $5,5 \pm 0,1^{\mathrm{b}}$ & $5,5 \pm 0,1^{\mathrm{b}}$ & $5,5 \pm 0,1^{\mathrm{b}}$ & $5,5 \pm 0,2^{\mathrm{b}}$ & $5,5 \pm 0,1^{\mathrm{b}}$ \\
\hline Protéines $(\%)$ & $1,4 \pm 0,01^{\mathrm{a}}$ & $1,5 \pm 0,01^{\mathrm{a}}$ & $1,5 \pm 0,01^{\mathrm{a}}$ & $1,5 \pm 0,02^{\mathrm{a}}$ & $1,5 \pm 0,01^{\mathrm{a}}$ & $1,5 \pm 0,01^{\mathrm{a}}$ \\
\hline Amidon $(\%)$ & $47,93 \pm 0,5^{\mathrm{a}}$ & $35,6 \pm 0,6^{\mathrm{b}}$ & $35,6 \pm 0,6^{\mathrm{b}}$ & $35,6 \pm 0,5^{\mathrm{b}}$ & $35,6 \pm 0,6^{\mathrm{b}}$ & $35,6 \pm 0,5^{b}$ \\
\hline Celluloses $(\%)$ & $2,1 \pm 0,1^{\mathrm{a}}$ & $0,25 \pm 0,01^{\mathrm{b}}$ & $0,25 \pm 0,01^{\mathrm{b}}$ & $0,25 \pm 0,01^{\mathrm{b}}$ & $0,25 \pm 0,01^{\mathrm{b}}$ & $0,25 \pm 0,01^{\mathrm{b}}$ \\
\hline Cendres $(\%)$ & $1,20 \pm 0,01^{\mathrm{a}}$ & $1,5 \pm 0,05^{\mathrm{a}}$ & $1,5 \pm 0,05^{\mathrm{a}}$ & $1,5 \pm 0,05^{\mathrm{a}}$ & $1,5 \pm 0,05^{\mathrm{a}}$ & $1,5 \pm 0,05^{\mathrm{a}}$ \\
\hline $\mathrm{pH}$ & $5,46 \pm 0,01^{\mathrm{a}}$ & $6,55 \pm 0,01^{\mathrm{b}}$ & $6,55 \pm 0,02^{\mathrm{b}}$ & $6,55 \pm 0,02^{\mathrm{b}}$ & $6,55 \pm 0,02^{\mathrm{b}}$ & $6,55 \pm 0,02^{\mathrm{b}}$ \\
\hline Acidité titrable (méq.g/100g) & $4,67 \pm 0,03^{\mathrm{a}}$ & $3,46 \pm 0,02^{\mathrm{b}}$ & $3,46 \pm 0,01^{\mathrm{b}}$ & $3,46 \pm 0,02^{\mathrm{b}}$ & $3,46 \pm 0,02^{\mathrm{b}}$ & $3,46 \pm 0,02^{\mathrm{b}}$ \\
\hline $\mathrm{HCN}(\mathrm{mg} / 100 \mathrm{~g})$ & 0,47 & - & - & - & - & - \\
\hline Energie $(\mathrm{kcal} / 100 \mathrm{~g})$ & $189,76 \pm 1,1^{\mathrm{a}}$ & $189,65 \pm 1,2^{\mathrm{a}}$ & $189,65 \pm 1,1^{\mathrm{a}}$ & $189,65 \pm 1,2^{\mathrm{a}}$ & $189,65 \pm 1,2^{\mathrm{a}}$ & $189,65 \pm 1,1^{\mathrm{a}}$ \\
\hline
\end{tabular}

Les valeurs sont les moyennes de 3 déterminations pour chaque critère et les valeurs portant les mêmes lettres dans la ligne ne sont pas significativement différentes au seuil de $5 \%$ selon le test de NewmanKeuls. 
Tableau 2 : Composition biochimique de l'attoukpou préparé avec le manioc frais et avec les granulés de manioc natif ( $\mathrm{g} / 100 \mathrm{~g}$ de matière sèche).

\begin{tabular}{|c|c|c|c|c|c|c|}
\hline Paramètres & $\begin{array}{l}\text { Attoukpou de } \\
\text { manioc frais }\end{array}$ & $\begin{array}{l}\text { Attoukpou de } \\
\text { granulés non } \\
\text { conservés }\end{array}$ & $\begin{array}{c}\text { Attoukpou de } \\
\text { granulés conservés } \\
\text { pendant } 6 \text { mois }\end{array}$ & $\begin{array}{c}\text { Attoukpou de } \\
\text { granulés conservés } \\
\text { pendant } 12 \text { mois }\end{array}$ & $\begin{array}{c}\text { Attoukpou de } \\
\text { granulés conservés } \\
\text { pendant } 18 \text { mois }\end{array}$ & $\begin{array}{c}\text { Attoukpou de } \\
\text { granulés conservés } \\
\text { pendant } 24 \text { mois }\end{array}$ \\
\hline Matières sèches (\%) & $48,2 \pm 0,90^{\mathrm{a}}$ & $48,85 \pm 0,9^{\mathrm{a}}$ & $48,85 \pm 0,8^{\mathrm{a}}$ & $48,85 \pm 0,7^{\mathrm{a}}$ & $48,85 \pm 0,9^{\mathrm{a}}$ & $48,85 \pm 0,8^{\mathrm{a}}$ \\
\hline Humidité (\%) & $51,8 \pm 0,85^{\mathrm{a}}$ & $51,15 \pm 0,7^{\mathrm{a}}$ & $51,15 \pm 0,6^{\mathrm{a}}$ & $51,15 \pm 0,5^{\mathrm{a}}$ & $51,15 \pm 0,8^{\mathrm{a}}$ & $51,15 \pm 0,7^{\mathrm{a}}$ \\
\hline Matières grasses $(\%)$ & $0,2 \pm 0,01^{\mathrm{a}}$ & $0,25 \pm 0,01^{\mathrm{a}}$ & $0,25 \pm 0,02^{\mathrm{a}}$ & $0,25 \pm 0,02^{\mathrm{a}}$ & $0,25 \pm 0,02^{\mathrm{a}}$ & $0,25 \pm 0,02^{\mathrm{a}}$ \\
\hline Glucides totaux (\%) & $49,13 \pm 0,7^{\mathrm{a}}$ & $49,5 \pm 0,6^{\mathrm{a}}$ & $49,5 \pm 0,5^{\mathrm{a}}$ & $49,5 \pm 0,5^{\mathrm{a}}$ & $49,5 \pm 0,6^{\mathrm{a}}$ & $49,5 \pm 0,5^{\mathrm{a}}$ \\
\hline Sucres totaux $(\%)$ & $0,8 \pm 0,01^{\mathrm{a}}$ & $8,4 \pm 0,1^{\mathrm{b}}$ & $8,4 \pm 0,1^{\mathrm{b}}$ & $8,4 \pm 0,2^{\mathrm{b}}$ & $8,4 \pm 0,1^{\mathrm{b}}$ & $8,4 \pm 0,2^{\mathrm{b}}$ \\
\hline Sucres réducteur $(\%)$ & $0,4 \pm 0,01^{\mathrm{a}}$ & $5,5 \pm 0,1^{\mathrm{b}}$ & $5,5 \pm 0,1^{\mathrm{b}}$ & $5,5 \pm 0,1^{\mathrm{b}}$ & $5,5 \pm 0,2^{\mathrm{b}}$ & $5,5 \pm 0,1^{\mathrm{b}}$ \\
\hline Protéines $(\%)$ & $1,4 \pm 0,01^{\mathrm{a}}$ & $1,5 \pm 0,01^{\mathrm{a}}$ & $1,5 \pm 0,01^{\mathrm{a}}$ & $1,5 \pm 0,02^{\mathrm{a}}$ & $1,5 \pm 0,01^{\mathrm{a}}$ & $1,5 \pm 0,01^{\mathrm{a}}$ \\
\hline Amidon (\%) & $47,93 \pm 0,5^{\mathrm{a}}$ & $35,6 \pm 0,6^{\mathrm{b}}$ & $35,6 \pm 0,6^{\mathrm{b}}$ & $35,6 \pm 0,5^{\mathrm{b}}$ & $35,6 \pm 0,6^{\mathrm{b}}$ & $35,6 \pm 0,5^{b}$ \\
\hline Celluloses $(\%)$ & $2,1 \pm 0,1^{\mathrm{a}}$ & $0,25 \pm 0,01^{\mathrm{b}}$ & $0,25 \pm 0,01^{\mathrm{b}}$ & $0,25 \pm 0,01^{\mathrm{b}}$ & $0,25 \pm 0,01^{\mathrm{b}}$ & $0,25 \pm 0,01^{\mathrm{b}}$ \\
\hline Cendres $(\%)$ & $1,20 \pm 0,01^{\mathrm{a}}$ & $1,5 \pm 0,05^{\mathrm{a}}$ & $1,5 \pm 0,05^{\mathrm{a}}$ & $1,5 \pm 0,05^{\mathrm{a}}$ & $1,5 \pm 0,05^{\mathrm{a}}$ & $1,5 \pm 0,05^{\mathrm{a}}$ \\
\hline $\mathrm{pH}$ & $5,46 \pm 0,01^{\mathrm{a}}$ & $6,55 \pm 0,01^{\mathrm{b}}$ & $6,55 \pm 0,02^{\mathrm{b}}$ & $6,55 \pm 0,02^{\mathrm{b}}$ & $6,55 \pm 0,02^{\mathrm{b}}$ & $6,55 \pm 0,02^{\mathrm{b}}$ \\
\hline Acidité titrable & $4,67 \pm 0,03^{\mathrm{a}}$ & $3,46 \pm 0,02^{\mathrm{b}}$ & $3,46 \pm 0,01^{\mathrm{b}}$ & $3,46 \pm 0,02^{\mathrm{b}}$ & $3,46 \pm 0,02^{\mathrm{b}}$ & $3,46 \pm 0,02^{\mathrm{b}}$ \\
\hline (méq.g/100g) & 0,47 & - & - & - & - & - \\
\hline $\mathrm{HCN}(\mathrm{mg} / 100 \mathrm{~g})$ & $189,76 \pm 1,1^{\mathrm{a}}$ & $189,65 \pm 1,2^{\mathrm{a}}$ & $189,65 \pm 1,1^{\mathrm{a}}$ & $189,65 \pm 1,2^{\mathrm{a}}$ & $189,65 \pm 1,2^{\mathrm{a}}$ & $189,65 \pm 1,1^{\mathrm{a}}$ \\
\hline Energie (Kcal/100g) & & & & & & \\
\hline
\end{tabular}


Tableau 3 : Composition biochimique du placali préparé avec le manioc frais et avec les granulés de manioc natif ( $\mathrm{g} / 100 \mathrm{~g}$ de matière sèche).

\begin{tabular}{|c|c|c|c|c|c|c|}
\hline Paramètres & $\begin{array}{l}\text { Placali de } \\
\text { Manioc } \\
\text { frais }\end{array}$ & $\begin{array}{l}\text { Placali de } \\
\text { granulés non } \\
\text { conservés }\end{array}$ & $\begin{array}{c}\text { Placali de } \\
\text { granulés conservés } \\
\text { pendant } 6 \text { mois }\end{array}$ & $\begin{array}{c}\text { Placali de granulés } \\
\text { conservés } \\
\text { pendant12 mois }\end{array}$ & $\begin{array}{c}\text { Placali de granulés } \\
\text { conservés } \\
\text { pendant } 18 \text { mois }\end{array}$ & $\begin{array}{c}\text { Placali de granulés } \\
\text { conservés pendant } \\
24 \text { mois }\end{array}$ \\
\hline Humidité (\%) & $69,9 \pm 0,3^{\mathrm{a}}$ & $69,9 \pm 0,3^{\mathrm{a}}$ & $70,1 \pm 0,2^{\mathrm{a}}$ & $70,1 \pm 0,1^{\mathrm{a}}$ & $70,2 \pm 0,2^{\mathrm{a}}$ & $70,1 \pm 0,1^{\mathrm{a}}$ \\
\hline Matières sèches (\%) & $30,1 \pm 0,1^{\mathrm{a}}$ & $30,1 \pm 0,1^{\mathrm{a}}$ & $29,9 \pm 0,1^{\mathrm{a}}$ & $29,9 \pm 0,1^{\mathrm{a}}$ & $29,8 \pm 0,1^{\mathrm{a}}$ & $29,9 \pm 0,1^{\mathrm{a}}$ \\
\hline Matières grasses $(\%)$ & 0 & 0 & 0 & 0 & 0 & 0 \\
\hline Glucides totaux $(\%)$ & $22,74 \pm 0,01^{\mathrm{a}}$ & $22,74 \pm 0,2^{\mathrm{a}}$ & $22,74 \pm 0,2^{\mathrm{a}}$ & $22,74 \pm 0,2^{\mathrm{a}}$ & $22,74 \pm 0,2^{\mathrm{a}}$ & $22,74 \pm 0,2^{\mathrm{a}}$ \\
\hline Sucres totaux $(\%)$ & $12,3 \pm 0,1^{\mathrm{a}}$ & $13,2 \pm 0,2^{\mathrm{a}}$ & $13,2 \pm 0,1^{\mathrm{a}}$ & $13,3 \pm 0,1^{\mathrm{a}}$ & $12,95 \pm 0,1^{\mathrm{a}}$ & $13,5 \pm 0,1^{\mathrm{a}}$ \\
\hline Sucres réducteur (\%) & $3,03 \pm 0,01^{\mathrm{a}}$ & $3,5 \pm 0,1^{\mathrm{a}}$ & $3,55 \pm 0,01^{\mathrm{a}}$ & $3,5 \pm 0,01^{\mathrm{a}}$ & $3,4 \pm 0,01^{\mathrm{a}}$ & $3,5 \pm 0,01^{\mathrm{a}}$ \\
\hline Protéines (\%) & $4,1 \pm 0,05^{\mathrm{a}}$ & $3,9 \pm 0,2^{\mathrm{a}}$ & $3,8 \pm 0,1^{\mathrm{a}}$ & $4,2 \pm 0,01^{\mathrm{a}}$ & $4,1 \pm 0,05^{\mathrm{a}}$ & $3,9 \pm 0,03^{\mathrm{a}}$ \\
\hline Amidon (\%) & $8,37 \pm 0,03^{\mathrm{a}}$ & $6,04 \pm 0,04^{b}$ & $5,99 \pm 0,03^{\mathrm{b}}$ & $5,94 \pm 0,04^{\mathrm{b}}$ & $6,39 \pm 0,04^{b}$ & $5,74 \pm 0,03^{\mathrm{b}}$ \\
\hline Celluloses (\%) & $1,1 \pm 0,01^{\mathrm{a}}$ & $1,1 \pm 0,01^{\mathrm{a}}$ & $1,1 \pm 0,01^{\mathrm{a}}$ & $1,1 \pm 0,01^{\mathrm{a}}$ & $1,1 \pm 0,01^{\mathrm{a}}$ & $1,1 \pm 0,01^{\mathrm{a}}$ \\
\hline Cendres $(\%)$ & $1,2 \pm 0,01^{\mathrm{a}}$ & $1,2 \pm 0,01^{\mathrm{a}}$ & $1,2 \pm 0,01^{\mathrm{a}}$ & $1,2 \pm 0,01^{\mathrm{a}}$ & $1,2 \pm 0,01^{\mathrm{a}}$ & $1,2 \pm 0,01^{\mathrm{a}}$ \\
\hline $\mathrm{pH}$ & $5,7 \pm 0,02^{\mathrm{a}}$ & $6,7 \pm 0,1^{\mathrm{a}}$ & $6,7 \pm 0,1^{\mathrm{a}}$ & $6,7 \pm 0,1^{\mathrm{a}}$ & $6,7 \pm 0,1^{\mathrm{a}}$ & $6,7 \pm 0,1^{\mathrm{a}}$ \\
\hline Acidité titrable (méq.g/100g) & $20 \pm 0,1^{\mathrm{a}}$ & $5 \pm 0,05^{\mathrm{b}}$ & $5 \pm 0,05^{\mathrm{b}}$ & $5 \pm 0,04^{b}$ & $4,5 \pm 0,03^{b}$ & $4,5 \pm 0,03^{b}$ \\
\hline $\mathrm{HCN}(\mathrm{mg} / 100 \mathrm{~g})$ & 0,9 & - & - & - & - & - \\
\hline Energie $(\mathrm{Kcal} / 100 \mathrm{~g})$ & $106,96 \pm 0,1^{\mathrm{a}}$ & $106,96 \pm 0,8^{\mathrm{a}}$ & $106,96 \pm 0,1^{\mathrm{a}}$ & $106,96 \pm 0,1^{\mathrm{a}}$ & $106,96 \pm 0,1^{\mathrm{a}}$ & $106,96 \pm 0,1^{\mathrm{a}}$ \\
\hline
\end{tabular}


Tableau 4 : Evaluation organoleptique de l'attiéké préparé avec le manioc frais et les granulés conservés pendant 24 mois à température ambiante.

\begin{tabular}{|c|c|c|c|c|c|c|}
\hline Paramètres & $\begin{array}{c}\text { Attiéké de } \\
\text { manioc frais }\end{array}$ & $\begin{array}{c}\text { Attiéké de } \\
\text { granulés } \\
\text { non } \\
\text { conservés }\end{array}$ & $\begin{array}{c}\text { Attiéké de } \\
\text { granulés } \\
\text { conservés } \\
\text { pendant } 6 \\
\text { mois }\end{array}$ & $\begin{array}{l}\text { Attiéké de } \\
\text { granulés } \\
\text { conservés } \\
\text { pendant } \\
12 \text { mois }\end{array}$ & $\begin{array}{c}\text { Attiéké de } \\
\text { granulés } \\
\text { conservés } \\
\text { pendant } \\
18 \text { mois }\end{array}$ & $\begin{array}{c}\text { Attiéké de } \\
\text { granulés } \\
\text { conservés } \\
\text { pendant } \\
24 \text { mois }\end{array}$ \\
\hline Couleur & $3,18 \pm 0,2^{\mathrm{a}}$ & $3,40 \pm 0,1^{\mathrm{a}}$ & $3,20 \pm 0,1^{\mathrm{a}}$ & $3,31 \pm 0,1^{\mathrm{a}}$ & $3,18 \pm 0,1^{\mathrm{a}}$ & $3,18 \pm 0,1^{\mathrm{a}}$ \\
\hline Arôme & $3,06 \pm 0,1^{\mathrm{a}}$ & $3,16 \pm 0,1^{\mathrm{a}}$ & $3,20 \pm 0,1^{\mathrm{a}}$ & $3,00 \pm 0,1^{\mathrm{a}}$ & $3,16 \pm 0,1^{\mathrm{a}}$ & $3,16 \pm 0,1^{\mathrm{a}}$ \\
\hline Goût/aigreur & $2,72 \pm 0,1^{a}$ & $3,90 \pm 0,2^{\mathrm{b}}$ & $3,86 \pm 0,2^{b}$ & $3,84 \pm 0,2^{\mathrm{b}}$ & $3,92 \pm 0,2^{b}$ & $3,92 \pm 0,2^{b}$ \\
\hline Texture & $3,54 \pm 0,2^{\mathrm{a}}$ & $3,36 \pm 0,1^{\mathrm{a}}$ & $3,54 \pm 0,1^{\mathrm{a}}$ & $3,40 \pm 0,1^{\mathrm{a}}$ & $3,32 \pm 0,1^{\mathrm{a}}$ & $3,32 \pm 0,1^{\mathrm{a}}$ \\
\hline
\end{tabular}

Tableau 5 : Evaluation organoleptique de l'attoukpou préparé avec le manioc frais et avec les granulés conservés pendant 24 mois à température ambiante.

\begin{tabular}{lcccccc}
\hline Paramètres & $\begin{array}{c}\text { Attoukpou } \\
\text { de manioc } \\
\text { frais }\end{array}$ & $\begin{array}{c}\text { Attoukpou } \\
\text { de granulés } \\
\text { non } \\
\text { conservé }\end{array}$ & $\begin{array}{c}\text { Attoukpou } \\
\text { de granulés } \\
\text { conservé } \\
\text { pendant } \mathbf{6} \\
\text { mois }\end{array}$ & $\begin{array}{c}\text { Attoukpou } \\
\text { de granulés } \\
\text { conservé } \\
\text { pendant } 12 \\
\text { mois }\end{array}$ & $\begin{array}{c}\text { Attoukpou } \\
\text { de granulés } \\
\text { conservé } \\
\text { pendant } \mathbf{1 8} \\
\text { mois }\end{array}$ & $\begin{array}{c}\text { Attoukpou } \\
\text { de granulés } \\
\text { conservé } \\
\text { pendant 24 } \\
\text { mois }\end{array}$ \\
\hline Couleur & $3,18 \pm 0,2^{\mathrm{a}}$ & $3,40 \pm 0,1^{\mathrm{a}}$ & $3,20 \pm 0,1^{\mathrm{a}}$ & $3,31 \pm 0,1^{\mathrm{a}}$ & $3,18 \pm 0,1^{\mathrm{a}}$ & $3,18 \pm 0,1^{\mathrm{a}}$ \\
Arôme & $3,06 \pm 0,1^{\mathrm{a}}$ & $3,16 \pm 0,1^{\mathrm{a}}$ & $3,20 \pm 0,1^{\mathrm{a}}$ & $3,00 \pm 0,1^{\mathrm{a}}$ & $3,16 \pm 0,1^{\mathrm{a}}$ & $3,16 \pm 0,1^{\mathrm{a}}$ \\
Goût/aigreur & $2,72 \pm 0,1^{\mathrm{a}}$ & $3,90 \pm 0,2^{\mathrm{b}}$ & $3,86 \pm 0,2^{\mathrm{b}}$ & $3,84 \pm 0,2^{\mathrm{b}}$ & $3,92 \pm 0,2^{\mathrm{b}}$ & $3,92 \pm 0,2^{\mathrm{b}}$ \\
Texture & $3,54 \pm 0,2^{\mathrm{a}}$ & $3,36 \pm 0,1^{\mathrm{a}}$ & $3,54 \pm 0,1^{\mathrm{a}}$ & $3,40 \pm 0,1^{\mathrm{a}}$ & $3,32 \pm 0,1^{\mathrm{a}}$ & $3,32 \pm 0,1^{\mathrm{a}}$ \\
\hline
\end{tabular}

Les valeurs sont les moyennes de 50 déterminations pour chaque critère et les valeurs portant les mêmes lettres dans chaque colonne ne sont pas significativement différentes au seuil de 5\% selon le test de Newman-Keuls.

Tableau 6 : Evaluation organoleptique du placali préparé avec le manioc frais et avec les granulés conservés pendant 24 mois à température ambiante.

\begin{tabular}{lcccccc}
\hline Paramètres & $\begin{array}{c}\text { placali de } \\
\text { manioc } \\
\text { frais }\end{array}$ & $\begin{array}{c}\text { placali de } \\
\text { granulés } \\
\text { non } \\
\text { conservés }\end{array}$ & $\begin{array}{c}\text { placali de } \\
\text { granulés } \\
\text { conservés } \\
\text { pendant } 6 \\
\text { mois }\end{array}$ & $\begin{array}{c}\text { placali de } \\
\text { granulés } \\
\text { conservés } \\
\text { pendant 12 } \\
\text { mois }\end{array}$ & $\begin{array}{c}\text { placali de } \\
\text { granulés } \\
\text { conservés } \\
\text { pendant 18 } \\
\text { mois }\end{array}$ & $\begin{array}{c}\text { placali de } \\
\text { granulés } \\
\text { conservés } \\
\text { pendant } \\
\text { 24 mois }\end{array}$ \\
\hline couleur & $3,44 \pm 0,1^{\mathrm{a}}$ & $3,22 \pm 0,1^{\mathrm{a}}$ & $3,58 \pm 0,1^{\mathrm{a}}$ & $3,26 \pm 0,1^{\mathrm{a}}$ & $3,22 \pm 0,1^{\mathrm{a}}$ & $3,22 \pm 0,1^{\mathrm{a}}$ \\
Arôme & $3,06 \pm 0,05^{\mathrm{a}}$ & $3,16 \pm 0,08^{\mathrm{a}}$ & $3,28 \pm 0,04^{\mathrm{a}}$ & $3,02 \pm 0,02^{\mathrm{a}}$ & $3,27 \pm 0,03^{\mathrm{a}}$ & $3,27 \pm 0,03^{\mathrm{a}}$ \\
Goût/aigreur & $2,68 \pm 0,05^{\mathrm{a}}$ & $3,60 \pm 0,08^{\mathrm{b}}$ & $3,72 \pm 0,05^{\mathrm{b}}$ & $3,60 \pm 0,05^{\mathrm{b}}$ & $3,60 \pm 0,05^{\mathrm{b}}$ & $3,60 \pm 0,05^{\mathrm{b}}$ \\
texture & $3,62 \pm 0,02^{\mathrm{a}}$ & $3,52 \pm 0,02^{\mathrm{a}}$ & $3,70 \pm 0,03^{\mathrm{a}}$ & $3,46 \pm 0,03^{\mathrm{a}}$ & $3,54 \pm 0,03^{\mathrm{a}}$ & $3,54 \pm 0,04^{\mathrm{a}}$ \\
\hline \multicolumn{2}{l}{$\begin{array}{l}\text { Les valeurs sont les moyennes de } 50 \text { déterminations pour chaque critère et les valeurs portant les mêmes lettres dans chaque } \\
\text { colonne ne sont pas significativement différentes au seuil de } 5 \% \text { selon le test de Newman-Keuls. }\end{array}$}
\end{tabular}




\section{DISCUSSION}

Trois cultivars de manioc (Bonoua, Yacé, Zoglo) ont été utilisés pour la production des granulés de manioc natif pouvant être conservés sur de longue période (Yao, 2015) puis reconstitués et utilisés dans la préparation les mets traditionnels tels que l'atiéké, l'attoukpou et le placali. Les produits obtenus, à partir des granulés conservés pendant 24 mois à température ambiante, sont exempts d'acide cyanhydrique et ont gardé la couleur des mêmes produits fabriqués avec le manioc frais après. Le manioc contient des polyphénols dont les plus importants sont la L-tyrosine, la dopamine, le pyrocatéchole et le pyrogallole. Ces composés qui sont responsables du brunissement enzymatique du manioc trois jours après la récolte comme l'ont montré Gogbeu et al. (2011), ont certainement été éliminés au cours $\mathrm{du}$ pressage du manioc broyé et le séchage rapide de la pâte pressée à plus de $40{ }^{\circ} \mathrm{C}$ a permis d'évaporer l'acide cyanhydrique (HCN) résiduel contenu dans la pâte de manioc. Les granulés ont été ainsi débarrassés de ce composé toxique. Ce résultat est en accord avec ceux de Hongbété et al. (2009) et Agbor-Egbe et Mbone (2006), qui ont montré que l'eau d'écoulement au cours du pressage du manioc broyé permet d'éliminer $90 \%$ de l'acide cyanhydrique et les polyphénols du manioc. Ainsi nous pensons que les $10 \%$ de $\mathrm{HCN}$ résiduel ont été éliminés au cours du séchage et de la fermentation subséquente au cours de la préparation de ces trois mets. Ces observations concordent également avec celles de Obilie et al. (2003) et Onwaka et Ogboga (2007) qui ont trouvé que la fermentation du manioc permet d'éliminer le HCN dans le fufu, qui est une pâte préparée avec la farine de manioc fermenté. La transformation du manioc en granulé de manioc natif peut être considérée comme une méthode simple et efficace pour éliminer l'acide cyanhydrique. Les produits obtenus après reconstitution des granulés de manioc natif et cuisson sont moins aigres que ceux préparés avec le manioc frais. Cela est certainement dû à l'élimination de $\mathrm{HCN}$ et des polyphénols, qui semblent servir d'intermédiaire lors de la fermentation, en favorisant la production d'acide organique ; ce qui a probablement entraîné l'augmentation de l'acidité et l'abaissement du $\mathrm{pH}$.

L'évaluation de la couleur des produits obtenus avec les granulés n'a pas donné de différence significative avec les produits faits avec le manioc frais, malgré le temps de conservation relativement long de 24 mois. Ce résultat était attendu, car, le traitement du manioc au cours de la production des granulés, qui a consisté à éplucher, broyer, presser et sécher le manioc dans les $48 \mathrm{~h}$ après la récolte n'a pas permis les réactions de brunissement enzymatique sous l'action des polyphénols de se produire comme l'ont montré Gogbeu et al. (2011).

Selon les résultats des tests de dégustation, l'attiéké, l'attoukpou et le placali préparés avec les granulés de manioc natif semblent avoir meilleur goût, comparativement aux produits de manioc frais. L'une des explications possibles semblent être d'une part, l'élimination des composés susceptibles de générer un arrière goût amer et, d'autre part, il y a probablement une hydrolyse partielle de l'amidon au cours de la cuisson libérant ainsi le glucose. Ces résultats confirment ceux de Padomou et al. (2005) qui ont observé le goût sucré du placali. En effet, le résultat des analyses biochimiques a montré que le taux d'amidon des granulés est inférieur à celui du manioc frais, alors que les valeurs des sucres totaux et des sucres réducteurs des granulés sont supérieures à celle du manioc frais. Le résultat des tests de dégustation confirme cette observation, car les trois produits préparés avec les granulés ont été jugés de meilleur goût, avec des différences significatives pour le paramètre goût.

Par ailleurs, il convient de souligner que le taux d'humidité de l'attoukpou est supérieur à celui de l'attiéké et cela, parce qu'au cours de la préparation, la semoule destinée à la confection de l'attoukpou n'est pas séchée mais est cuite, immédiatement, après le pressage et l'émottage ; ce qui permet 
d'obtenir le disque de l'attoukpou en un seul bloc. Contrairement à l'attoukpou, la semoule utilisée pour préparer l'attiéké est granulée, puis, partiellement séchée, pour réduire le taux d'humidité. Il s'en suit que lors de la cuisson, les grains d'attiéké se détachent et donnent un produit semblable à la semoule de blé, communément appelée couscous (KoffiNevry et al., 2007).

\section{Conclusion}

Les granulés de manioc natif conservés pendant deux ans ont été reconstitués pour obtenir l'équivalent de manioc frais broyé puis utilisés pour préparer de manière satisfaisante les mets traditionnels ivoiriens : l'attiéké, l'attoukpou et le placali. Les produits obtenus présentent des propriétés biochimiques et organoleptiques très intéressantes comparativement à ceux obtenus avec le manioc fraîchement récolté. Ainsi, les mets issus des granulés reconstitués sont totalement dépourvus d'acide cyanhydrique et ont été jugés de meilleur goût.

\section{REFERENCES}

Abbor-Egbe T, Mbone IL. 2006. The effect of processing techniques in reducing cyanogen levels during production of some Cameroonian cassava food. $J$. Food Compos. Anal., 19: 354-363.

Anonyme. 2008. Ministère de l'agriculture : concertation régionale sur la situation alimentaire et nutritionnelle au sahel et en Afrique de l'ouest. Rapport Côte d'Ivoire, $21 \mathrm{p}$.

AOAC. 2000. Official Methods of Analysis $\left(13^{\text {th }}\right.$ edn). Association of Official Analytical Chemist: Washington, D C.

Atwater W, Rosa E. 1899. A new respiratory colorimeter and the conservation of energy in human body. Physiol. Rev., 9: 214-251.

Bernfeld P. 1955. Amylase and Proteases. In Methods in Enzymology, Colswick SP, Kaplan NO (eds). Academic Press: New-York, USA; 149-154.
Bertrand G, Thomas P. 1910. Guide pour les Manipulations de Chimie Biologie. Dunod : Paris.

BIPEA. 1976. Recueil des Méthodes d'Analyse des Communautés Européennes. BIPEA: Genevilliers; 51-52.

Coleman CH. 1970. Calculations used in food analysis, In IFT World Directory guide. Publication of the Institute of Food Technologists: Chicago, Illinois USA; 326-331.

Dubois M, Gilles KA, Hamilton JK, Rebers PA and Smith F. 1956. Colorimetric method for determination of sugars and related substances. Anal. Chem., 28: 350-356.

Ducroquet H. 2002. L'agriculture ivoirienne à la loupe (1). Profes. agr., 3: 10 -12.

FAO. 2009a. Agricultural Statistics. Food and Agriculture Organization of the United Nations: Rome.

FAO. 2009b. Agricultural Statistics. Food and Agriculture Organization of the United Nations: Rome.

FAO. 2008. Corporate Document Repository: The impact of HIV/AIDS on The agricultural sector, http//www.fao.org/ docrep/005//Y4636E/y4636e05.htm.

Gogbeu JS, Dogbo DO, Gonnety TJ, N'zué B, Zohouri GP, Boka A. 2011. Study of some characteristics of soluble polyphenol oxidases from six cultivars callus off cassava (Manihot esculenta Crantz). J. An. Plant Sci., 9: 11691179.

Hongbété F, Mestres C, Akissoé N, Nago MC. 2009. Effect of processing conditions on cyanide content and colour of cassava flours from West Africa. African Journal of Food Science, 33(1): 001-006.

Koffi-Nevry R, Koussemou M, Aboua F. 2007. Chemical and organoleptic properties of attoukpou made from two cassava varieties (Manihot Esculenta Crantz), Bonoua and IAC. J. Food Technol., 54: 300-304. 
Liebig-Denige. 1971. Dosage de l'Acide Cyanhydrique. Meded. Landbouw Hogeschool: Wageningen 71, 13p.

N'Zué B, Zohouri PG et Sangaré A: 2004. Performance agronomique de quelques variétés de manioc de trois zones agro climatiques de la Côte d'Ivoire. Agron. Afr., 16: 1-7.

Obilie EM, Tanoh-Debrah K, Amoa-Awua. 2003. Microbial modification of the texture of grated cassava during fermentation into akyeke. Int. J. Food Microbiol., 89: 275-280.

Obadina AO, Oyewole OB, Odusami AO. 2009. Microbiological safety and quality Assessment of some fermented cassava products (lafu, fufu, gari). Scient Res Ess., 4(5): 432-435.

Onwaka GI, Ogbogu NJ. 2007. Effect of fermentation on the quality and physicochemical properties of cassava based Fufu products made from two cassava varieties NR8212 and Nwangbisi. Medwell J. Food Tech., 5: 261-264.
Padonou W, Mestres C, Nago CM. 2005. The quality of boiled cassava: instrumental characterization and relationship with physic-chemical properties and sensorial properties. Food Chem., 89: 261-270.

Udedible ABI, Enyenihi GE, Akpan MJ, Obasi OL, Solomon IP. 2009. Physical nature and nutritive value of dried cassava $F u f u$ meal for laying hens. Niger. Agric. J., 39: 44-49.

Yao KA. 2015. Contribution à la réduction des pertes post-récoltes de la banane plantain (Musa $A A B$ ) et du manioc (Manihot esculenta Crantz) par des approches technologiques de conservation et de transformation. Thèse de Doctorat Unique de l'Université Félix Houphöuet-Boigny (Abidjan, Côte d'Ivoire), 158p.

Yao KJ, Kofi RA, Aboua F. 2006. Composition of dehydrated attiéké powder. Trop. Sci., 46: 224-226. 